\title{
Analysis of a Mathematical Model to Investigate the Dynamics of Dengue Fever
}

\section{${ }^{* 1}$ ANDRAWUS JAMES; EGUDA, FELIX YAKUBU}

\author{
${ }^{1}$ Department of Mathematics, Federal University, Dutse, Jigawa State \\ E-mail-felyak_e@yahoo.co.uk
}

\begin{abstract}
In this paper, we formulated a compartmental model to investigate the dynamics of dengue fever in a population with some measure of disease control. We qualitatively and quantitatively analyzed the model and found that the model has a disease free equilibrium (DFE), an endemic equilibrium point and undergoes the phenomenon of backward bifurcation. It was also discovered that Dengue can be eliminated irrespective of the initial size of the infected population whenever the effective reproduction number is less than one. Numerical simulations were carried out on the model and effective control measures were proposed that will result in reducing the burden of the disease in the population. ( ) JASEM
\end{abstract}

\section{https://dx.doi.org/10.4314/jasem.v21i4.2}

Keywords: Dengue Fever, Mathematical Model, Equilibrium, Bifurcation analysis, Effective reproduction number, Stability.

Dengue, a mosquito-transmitted disease caused by any of four closely-related virus serotypes (DEN-1-4) of the genus Flavivirus, is endemic in at least 100 countries in Africa, the Americas, the Eastern Mediterranean and subtropical regions of the world, inhibited by over 2.5 billion people (Garba, et al., 2008). In developing countries population growth is an important factor that contributes to the increase in the incidence of communicable diseases which affects mainly the urban poor, with infants and children among the groups particularly at risk (Nuraini et al, 2009). Urbanization and population growth increase the demand on the basic essential services such as housing, water supply, etc., and at the same time induce conditions that increase the transmission of some vector-borne diseases (Nuraini et al., 2009). Dengue is a viral, vector borne disease, spread by the Aedes Aegypti mosquito. It was estimated that about 50 million infections occur annually in over 100 countries. There is no specific treatment for curing dengue patients (Nuraini et al., 2009). Hospital treatment, in general, is given as supportive care which includes bed rest and analgesics (Nuraini et al., 2009).

Dengue virus is one of the most difficult arboviruses to isolate (Nuraini et al., 2009). There are four serotypes of the dengue virus; Den-1, Den-2, Den-3, Den-4, and each of the serotypes has numerous virus strains (Nuraini et al., 2009). Infection with one dengue serotypes may provide long life immunity to that serotype, but there is no complete crossprotective immunity to other serotype (Gubler, 1998). Identification of the primary target cells of dengue viruses' replication in the infected human body has proven to be extremely difficult (Nuraini et al., 2009).

The incubation period of the disease in an infected host is 3-14 days (average 4-7 days) (Nuraini et al., 2009). At the end of the incubation period, the patient may experience a sudden onset of fever (Nuraini et $a l, 2009)$. Viraemia is the presence of the virus in the blood stream (Nuraini et al., 2009). It is detected using the mosquito inoculation technique. Viraemia is assumed to become detectable on the second or the third day before the onset of symptoms and ends on the last days of illness (Nuraini et al., 2009). It usually peaks at the time of or shortly after the onset of illness (Gubler et al., 1981). Susceptible mosquitoes can be infected when they bite dengue infected hosts during the febrile viremic stage (Nuraini et al., 2009). It is usually believed that dengue viruses quickly clear in human body within approximately 7 days after the day of sudden onset of fever (Vaughn et al., 1994). Naturally this clearing process is done by the immune system which is as a result of complex dynamics reactions (Nuraini et al., 2009). Over the last decade mathematical models have been formulated to evaluate the dynamics of Dengue Fever. In this paper, a mathematical model is formulated and analysed to investigate the dynamics of Dengue Fever in a population in order to reduce the public health burden of the disease.

\section{MATERIALS AND METHODS}

Let $N_{H}(t)$ and $N_{V}(t)$ denote the total number of humans and vectors at time $t$, respectively. The model sub-divides these populations into a number of mutually-exclusive compartments, as given below. 
The total population of human and vectors is divided into the following mutually exclusive epidemiological classes, namely, susceptible humans $\left(S_{H}(t)\right)$, humans with dengue in latent stage $\left(E_{1}(t)\right)$, humans with dengue $\left(\mathrm{I}_{\mathrm{I}}(\mathrm{t})\right)$, humans treated of dengue $\left(\mathrm{R}_{1}(\mathrm{t})\right)$, susceptible vectors $\left(\mathrm{S}_{\mathrm{V}}(\mathrm{t})\right)$, vectors with latent dengue $\left(E_{V}(t)\right)$, vectors with dengue $\left(I_{V}(t)\right)$, Hence, we have that,

$$
\begin{aligned}
& N_{H}(t)=S_{H}(t)+E_{1}(t)+I_{1}(t)+R_{1}(t) \\
& \text { and } \\
& N_{V}(t)=S_{V}(t)+E_{V}(t)+I_{V}(t)
\end{aligned}
$$

Susceptible humans are recruited at a rate $\Lambda_{H}$ while the susceptible vectors are recruited at a rate $\Lambda_{V}$. Susceptible humans contract dengue at a rate

$$
\lambda_{D V}=\frac{\beta_{V H}\left(\eta_{v} E_{v}+I_{v}\right)}{N_{H}}
$$

where $\eta_{v}<1$, this accounts for the relative infectiousness of vectors with latent dengue $\mathrm{E}_{\mathrm{V}}$ compared to vectors in the $\mathrm{I}_{\mathrm{V}}$ class.

Susceptible vectors acquire dengue infection from infected humans at a rate

$\lambda_{D H}=\frac{\beta_{H V}\left(\eta_{A} E_{1}+\eta_{B} I_{1}\right)}{N_{H}}$,

Where $\eta_{A}<\eta_{B}$, this accounts for the relative infectiousness of humans with latent dengue $\mathrm{E}_{1}$ compared to humans in the $\mathrm{I}_{1}$ class.

Derivation of Model Equations: Singly infected individuals with latent dengue progress to active dengue at a rate $\gamma_{1}$. Natural human death occurs at a rate $\mu_{H}$ in the classes $S_{H \prime}, E_{1}, I_{1}, R_{1}$, respectively and those in $I_{1}$ class undergo an additional dengue induced death, at rate $\delta_{D 1}$. Natural vector death occurs, at a rate $\mu_{V}$, in the classes $S_{V}, E_{V}$ and $I_{V}$, while the vectors in the $I_{V}$ class undergoes additional dengue induced death, at a rate $\delta_{H V}$, although this is negligible as infected vectors are not deemed to be suffering dengue. Exposed vectors progress to the infectious stage at the rate $\gamma_{V}$.

The above assumptions result in the following system of nonlinear ordinary differential equations:

$$
\begin{aligned}
& \dot{S}_{H}=\Lambda_{H}-\mu_{H} S_{H}-\lambda_{D V} S_{H}, \\
& \dot{E}_{1}=\lambda_{D V} S_{H}-\left(\gamma_{1}+\mu_{H}\right) E_{1}, \\
& \dot{I}_{1}=\gamma_{1} E_{1}-\left(\tau_{1}+\mu_{H}+\delta_{D 1}\right) I_{1}, \\
& \dot{R}_{1}=\tau_{1} I_{1}-\mu_{H} R_{1} \\
& \dot{S}_{V}=\Lambda_{V}-\lambda_{D H} S_{V}-\mu_{V} S_{V}, \\
& \dot{E}_{V}=\lambda_{D H} S_{V}-\left(\gamma_{V}+\mu_{V}\right) E_{V}, \\
& \dot{I}_{V}=\gamma_{V} E_{V}-\left(\mu_{V}+\delta_{H V}\right) I_{V},
\end{aligned}
$$

Table 1: Description of the state variables of the model 1

\begin{tabular}{ll}
\hline \multicolumn{1}{c}{ Variable } & \multicolumn{1}{c}{ Description } \\
\hline $\boldsymbol{S}_{H}$ & Susceptible human population \\
$\boldsymbol{E}_{\boldsymbol{I}}$ & Human population with dengue in latent stage \\
$\boldsymbol{I}_{\boldsymbol{I}}$ & Human population with dengue (Dengue only) \\
$\boldsymbol{R}_{\boldsymbol{I}}$ & Human population treated of dengue (Dengue only) \\
$\boldsymbol{S}_{V}$ & Susceptible vectors population \\
$\boldsymbol{E}_{V}$ & Exposed vectors \\
$\boldsymbol{I}_{V}$ & Infectious vectors \\
\hline
\end{tabular}


Table 2: Description of Parameters of the Model (1)

\begin{tabular}{|c|c|c|c|c|}
\hline Parameter & Description & Values & Unit & Reference \\
\hline$\Lambda_{H}, \Lambda_{V}$ & $\begin{array}{l}\text { Recruitment rate into the population of } \\
\text { susceptible } \\
\begin{array}{ll}\text { respectively. } & \text { humans, vectors } \\
\end{array}\end{array}$ & 500,10000000 & Year $^{-1}$ & Garba et al, 2008. \\
\hline $\boldsymbol{\mu}_{H}, \boldsymbol{\mu}_{v}$ & $\begin{array}{l}\text { Natural death for humans, vectors } \\
\text { respectively. }\end{array}$ & $0.02041,36.5$ & Year $^{-1}$ & Okuonghae and Omosigho (2011). \\
\hline$\beta_{V H}$ & $\begin{array}{l}\text { Effective contact rate for dengue from } \\
\text { vectors to humans }\end{array}$ & 5 & $\begin{array}{l}\text { Year }^{-1} \\
\text { Year }^{-1}\end{array}$ & Garba et al, 2008. \\
\hline$\beta_{H V}$ & $\begin{array}{l}\text { Effective contact rate for dengue from } \\
\text { humans to vectors }\end{array}$ & 4 & Year $^{-1}$ & Garba et al, 2008. \\
\hline$\tau_{1}, \tau_{2}$ & Dengue treatment rate for $I_{l}, E_{l}$. & $2.5,1.5$ & Ind $^{-1}$ Year $^{-1}$ & Garba et al, 2008. \\
\hline$\gamma_{1}$ & Progression rate to active dengue & 0.3254 & Year $^{-1}$ & Garba et al, 2008. \\
\hline$\gamma_{V}$ & $\begin{array}{l}\text { Progression rate to active dengue } \\
\text { (vectors) }\end{array}$ & 0.03 & Year $^{-1}$ & Garba et al, 2008. \\
\hline & Disease induced death Dengue & 0.365 & Year $^{-1}$ & Okuonghae and Omosigho, (2011). \\
\hline $\begin{array}{l}\boldsymbol{\delta}_{\boldsymbol{D 1}} \\
\boldsymbol{\delta}_{H V}\end{array}$ & Disease induced death dengue (vectors) & 0 & Year $^{-1}$ & Garba et al, 2008. \\
\hline $\boldsymbol{k}_{V}$ & $\begin{array}{l}\text { Progression rate to active dengue } \\
\text { (vectors) }\end{array}$ & 0.05 & Year $^{-1}$ & Garba et al, 2008 . \\
\hline $\begin{array}{l}\boldsymbol{\eta}_{V} \\
\boldsymbol{\eta}_{A}, \boldsymbol{\eta}_{B}\end{array}$ & Modification parameters for $E_{v}, E_{l}, I_{l}$ & $\begin{array}{l}0.4,1.2,0.5,0.6,1,0.6,1 \\
.1,1,\end{array}$ & Year $^{-1}$ & Okuonghae and Omosigho, (2011) \\
\hline $\mathbf{P}_{\text {D1 }}$ & $\begin{array}{l}\text { Fraction of newly infected humans with } \\
\text { latent dengue }\end{array}$ & 0.6 & Year $^{-1}$ & Garba et al, 2008. \\
\hline
\end{tabular}

Consider the region $D_{2}=\left\{\left(S_{H}, E_{1}, I_{1}, R_{1}, S_{V}, E_{V}, I_{V}\right) \in \mathbb{R}_{+}^{7}: N_{H} \leq \frac{\Lambda_{H}}{\mu_{H}}, N_{V} \leq \frac{\Lambda_{V}}{\mu_{V}}\right\}$. It can be shown that the set $\mathrm{D}_{2}$ is positively invariant and an attractor of all positive solution of the system (1).

Lemma 1 The region $D_{2}$ is positively invariant for the system (1)

Proof: The rate of change of the total human population is given as

$\dot{N}_{H}=\dot{S}_{H}+\dot{E}_{1}+\dot{I}_{1}+\dot{R}_{1}=\Lambda_{H}-\mu_{H} N_{H}-\delta_{D 1} I_{1}$

By standard comparison theorem, $\dot{N}_{H} \leq \Lambda_{H}-\mu_{H} N_{H}$

So we have $\dot{N}_{H}+\mu_{H} N_{H} \leq \Lambda_{H}$.

Using the integrating factor method

$\dot{N}_{H} e^{\mu_{H} t}+\mu_{H} N_{H} e^{\mu_{H} t} \leq \Lambda_{H} e^{\mu_{H} t}$

$\frac{d}{d t}\left(\dot{N}_{H} e^{\mu_{H} t}\right) \leq \Lambda_{H} e^{\mu_{H} t}$

$\int d\left(\dot{N}_{H} e^{\mu_{H} t}\right) \leq \int \Lambda_{H} e^{\mu_{H} t} d t$

$N_{H} e^{\mu_{H} t} \leq \frac{\Lambda_{H}}{\mu_{H}} e^{\mu_{H} t}+D$

at $t=0, D=N_{H}(0)-\frac{\Lambda_{H}}{\mu_{H}}$

$N_{H} e^{\mu_{H} t} \leq \frac{\Lambda_{H}}{\mu_{H}} e^{\mu_{H} t}+N_{H}(0)-\frac{\Lambda_{H}}{\mu_{H}}$ 
$N_{H}=N_{H}(0) e^{-\mu_{H} t}+\frac{\Lambda_{H}}{\mu_{H}}\left[1-e^{-\mu_{H} t}\right]$

and the rate of change of the total vector population

$\dot{N}_{V}=\Lambda_{V}-\mu_{V} N_{V}-\delta_{H V} I_{V}$

By standard comparison theorem,

$\dot{N}_{V} \leq \Lambda_{V}-\mu_{V} N_{V}$

Similarly, using the integrating factor method, we have

$N_{V}=N_{V}(0) e^{-\mu_{V} t}+\frac{\Lambda_{V}}{\mu_{V}}\left[1-e^{-\mu_{V} t}\right]$

In particular $N_{H}(t) \leq \frac{\Lambda_{H}}{\mu_{H}}$ if $N_{H}(0) \leq \frac{\Lambda_{H}}{\mu_{H}}$ and $N_{V}(t) \leq \frac{\Lambda_{V}}{\mu_{V}}$ if $N_{V}(0) \leq \frac{\Lambda_{V}}{\mu_{V}}$, respectively.

So, $D_{2}$ is a positively invariant set under the flow described in (1). Hence, no solution path leaves through the boundary of $D_{2}$. Also, since solution paths cannot leave $D_{2}$, solutions remain non-negative for non-negative initial conditions. Solutions exist for all time t. In this region, the model (1) is said to be well posed mathematically and epidemiologically.

Positivity of Solutions

Lemma 2.Let the initial data for the model (1) be $S_{H}(t)>0, E_{1}(t)>0, I_{1}(t)>0, R_{1}(t)>0, S_{V}(t)>$ $0, E_{V}(t)$ and $I_{V}(t)>0$ then the solution $S_{H}(t), E_{1}(t), I_{1}(t), R_{1}(t), S_{V}(t), E_{V}(t)$, and $I_{V}(t)$ with positive initial data will remain positive for all time $t>0$.

Proof: Let $t_{1}=\sup \left\{t>0: S_{H}(t)>0, E_{1}(t)>0, I_{1}(t)>0, R_{1}(t)>0, S_{V}(t)>0, E_{V}(t)>0, I_{V}(t)>0\right\}>0$

$\dot{S}_{H}=\Lambda_{H}-\lambda_{D V} S_{H}-\mu_{H} S_{H}=\Lambda_{H}-\left(\lambda_{D V}+\mu_{H}\right) S_{H}$

To solve the ODE using the integrating factor method

I.F $=\exp \left[\mu_{H} t+\left\{\int_{0}^{t} \lambda_{D V}(\tau) d(\tau)\right\}\right]$

$\frac{d}{d t}\left[S_{H}(t) \exp \left\{\mu_{H} t+\int_{0}^{t} \lambda_{D V}(\tau) d(\tau)\right\}\right]=\Lambda_{H}\left[\exp \left\{\mu_{H} t+\int_{0}^{t} \lambda_{D V}(\tau) d(\tau)\right\}\right]$

$S_{H}\left(t_{1}\right) \exp \left\{\mu_{H} t_{1}+\int_{0}^{t_{1}} \lambda_{D V}(\tau) d(\tau)\right\}=S_{H}(0)+\int_{0}^{t_{1}} \Lambda_{H}\left[\exp \left\{\mu_{H} y+\int_{0}^{y} \lambda_{D V}(\tau) d(\tau)\right\}\right] d y$

$S_{H}\left(t_{1}\right)=S_{H}(0) \exp \left\{-\mu_{H} t_{1}-\int_{0}^{t_{1}} \lambda_{D V}(\tau) d(\tau)\right\}$

$+\left[\exp \left\{-\mu_{H} t_{1}-\int_{0}^{t_{1}} \lambda_{D V}(\tau) d(\tau)\right\}\right] \int_{0}^{t_{1}} \Lambda_{H}\left[\exp \left\{\mu_{H} y+\int_{0}^{y} \lambda_{D V}(\tau) d(\tau)\right\}\right] d y>0$

for $\dot{E}_{1}=\lambda_{D V} S_{H}-\left(\gamma_{1}+\mu_{H}\right) E_{1}$ we have that $\dot{E}_{T} \geq-\left(\gamma_{1}+\mu_{H}\right) E_{1}$,

for $\dot{I}_{1}=\gamma_{1} E_{1}-\left(\tau_{1}+\mu_{H}+\delta_{D 1}\right) I_{1} \quad$ we have that $\dot{I}_{1} \geq-\left(\tau_{1}+\mu_{H}+\delta_{D 1}\right) I_{1}$,

for $\dot{R}_{1}=\tau_{1} I_{1}-\mu_{H} R_{1}$ we have that $\dot{R}_{1} \geq-\mu_{H} R_{1}$,

for $\dot{S}_{V}=\Lambda_{V}-\lambda_{D H} S_{V}-\mu_{V} S_{V}$ we have that $\dot{S}_{V} \geq-\left(\lambda_{D H}+\mu_{V}\right) S_{V}$, 
for $\dot{E}_{V}=\lambda_{D H} S_{V}-\left(\gamma_{V}+\mu_{V}\right) E_{V}$ we have that $\dot{E}_{V} \geq-\left(\gamma_{V}+\mu_{V}\right) E_{V}$,

for $\dot{I}_{V}=\gamma_{V} E_{V}-\left(\mu_{V}+\delta_{H V}\right) I_{V}$ we have that $\dot{I}_{V} \geq-\left(\mu_{V}+\delta_{H V}\right) I_{V}$.

Similarly, we can show that $S_{H}(t)>0, E_{1}(t)>0, I_{1}(t)>0, R_{1}(t)>0, S_{V}(t)>0, E_{V}(t)>0$, and $I_{V}(t)>0$.

Local Stability of Disease-Free Equilibrium (DFE) of the Model: The model (1) has a disease-free equilibrium, obtained by setting the right hand side of the model to zero and also setting the disease classes to zero we obtain

$$
\xi_{2}=\left(S_{H}^{*}, E_{1}^{*}, I_{1}^{*}, R_{1}^{*}, S_{v}^{*}, E_{v}^{*}, I_{v}^{*}\right)=\left(\frac{\Lambda_{H}}{\mu_{H}}, 0,0,0, \frac{\Lambda_{v}}{\mu_{v}}, 0,0\right)
$$

The stability of $\xi_{2}$ is established using the next generation operator method on the system (1). Using the notation in van den Driessche and Watmough (2002) the matrices $F_{1}$ and $V_{1}$ for the new infection terms and the remaining transfer terms, are respectively given as

$F_{1}=\left(\begin{array}{cccc}0 & 0 & \beta_{V H} \eta_{V} & \beta_{V H} \\ 0 & 0 & 0 & 0 \\ \frac{\beta_{H V} \eta_{A} S_{V}}{N_{H}} & \frac{\beta_{H V} \eta_{B} S_{V}}{N_{H}} & 0 & 0 \\ 0 & 0 & 0 & 0\end{array}\right)$

And,

$V_{1}=\left(\begin{array}{cccc}g_{3} & 0 & 0 & 0 \\ -\gamma_{1} & g_{4} & 0 & 0 \\ 0 & 0 & g_{5} & 0 \\ 0 & 0 & -\gamma_{V} & g_{6}\end{array}\right)$

Where, $g_{3}=\mu_{H}+\gamma_{1}, g_{4}=\tau_{1}+\mu_{H}+\delta_{D 1}, g_{5}=\gamma_{V}+\mu_{V}, g_{6}=\mu_{V}+\delta_{H V}$

The spectral radius given by $\rho\left(F_{1} V_{1}^{-1}\right)=\sqrt{\frac{\Lambda_{V} \beta_{H V} \beta_{V H} \mu_{H}\left(g_{4} \eta_{A}+\gamma_{1} \eta_{B}\right)\left(\gamma_{V}+g_{6} \eta_{V}\right)}{\Lambda_{H} g_{3} g_{4} g_{5} g_{6} \mu_{V}}}=R_{D}$

The value $R_{D}$ is the effective reproduction number.

Lemma 3 The DFE of the system (1) is locally asymptotically stable if $R_{D}<1$ and unstable if $R_{D}>1$.

The threshold quantity $R_{D}$ is the effective or control reproduction number for the Dengue model. By Lemma 3, biologically speaking, Dengue is eliminated from the population when $R_{D}<1$ if the initial sizes of the subpopulations of the model are in the region of attraction of $\xi_{2}$.

However, the disease free equilibrium may not be globally asymptotically stable even if $R_{D}<1$ in the case when a backward bifurcation occurs. That is, there is the presence of a stable EEP co-existing with the DFE.

Existence of Endemic Equilibrium Point (EEP) of the model

Let the EEP of model (1) be denoted by $\xi_{(1, D)}=\left(S_{H}^{* *}, E_{1}^{* *}, I_{1}^{* *}, R_{1}^{* *}, S_{V}^{* *}, E_{V}^{* *}, I_{V}^{* *}\right)$. The equations in (1) are solved in terms of the force of infection at steady state and they are given as

$$
\begin{aligned}
& S_{H}^{* *}=\frac{\Lambda_{H}}{\mu_{H}+\lambda_{D V}^{* *}}, \\
& E_{1}^{* *}=\frac{\Lambda_{H} \lambda_{D V}^{* *}}{\left(\mu_{H}+\lambda_{D V}^{* *}\right)\left(\gamma_{1}+\mu_{H}\right)}, \\
& I_{1}^{* *}=\frac{\gamma_{1} \lambda_{D V}^{* *} \Lambda_{H}}{\left(\mu_{H}+\lambda_{D V}^{* *}\right)\left(\gamma_{1}+\mu_{H}\right)\left(\tau_{1}+\mu_{H}+\delta_{D 1}\right)},
\end{aligned}
$$




$$
\begin{aligned}
R_{1}^{* *} & =\frac{\tau_{1} \gamma_{1} \lambda_{D V}^{* *} \Lambda_{H}}{\left(\mu_{H}+\lambda_{D V}^{* *}\right)\left(\gamma_{1}+\mu_{H}\right)\left(\tau_{1}+\mu_{H}+\delta_{D 1}\right) \mu_{H}} \\
S_{V}^{* *} & =\frac{\Lambda_{V}}{\left(\mu_{V}+\lambda_{D H}^{* *}\right)}, \\
E_{V}^{* *} & =\frac{\lambda_{D H}^{* *} \Lambda_{V}}{\left(\mu_{V}+\lambda_{D H}^{* *}\right)\left(\gamma_{V}+\mu_{V}\right)}, \\
I_{V}^{* * *}= & \frac{\gamma_{V} \lambda_{D H}^{* *} \Lambda_{V}}{\left(\mu_{V}+\lambda_{D H}^{* *}\right)\left(\gamma_{V}+\mu_{V}\right)\left(\mu_{V}+\delta_{H V}\right)}, \\
N_{H}^{* *}= & \frac{\Lambda_{H} \mu_{H}\left(\gamma_{1}+\mu_{H}\right)\left(\tau_{1}+\mu_{H}+\delta_{D 1}\right)+\lambda_{D V}^{* *} \Lambda_{H}\left(\tau_{1}+\mu_{H}+\delta_{D 1}\right) \mu_{H}+}{\gamma_{1}\left(\lambda_{D V}^{* *} \Lambda_{H} \mu_{H}+\tau_{1} \gamma_{1} \lambda_{D V}^{* *} \Lambda_{H}\right.}
\end{aligned}
$$

now,

$\lambda_{D H}^{* *}=\frac{\beta_{H V}\left(\eta_{A} E_{1}^{* *}+\eta_{B} I_{1}^{* *}\right)}{N_{H}^{* *}}$ and $\lambda_{D V}^{* *}=\frac{\beta_{V H}\left(\eta_{V} E_{V}^{* *}+I_{V}^{* *}\right)}{N_{H}^{* *}}$

Substituting the values of $E_{1}^{* *}, I_{1}^{* *}, N_{H}^{* *}$ to $\lambda_{D H}^{* *}$ and $E_{V}^{* *}, I_{V}^{* *}, N_{H}^{* *}$ to $\lambda_{D V}^{* *}$ we have

$$
\lambda_{D H}^{* *}=\frac{\beta_{H V} \mu_{H}\left(g_{4} \eta_{A}+\gamma_{1} \eta_{B}\right) \lambda_{D V}^{* *}}{\mu_{H} g_{3} g_{4}+\left(\mu_{H} g_{4}+\gamma_{1} \mu_{H}+\tau_{1} \gamma_{1}\right) \lambda_{D V}^{* *}}
$$

and

$$
\begin{aligned}
\lambda_{D V}^{* *}= & \frac{\beta_{V H} \Lambda_{V} \mu_{H}\left(\eta_{V} g_{6}+\gamma_{V}\right) g_{3} g_{4}\left(\mu_{H}+\lambda_{D V}^{* *}\right) \lambda_{D H}^{* *}}{\Lambda_{H} \mu_{H} g_{3} g_{4} g_{5} g_{6} \mu_{V}+\left(\Lambda_{H} \mu_{H} g_{4} g_{5} g_{6} \mu_{H}+\left(\Lambda_{H} \gamma_{1} \mu_{H}+\tau_{1} \gamma_{1} \Lambda_{H}\right) g_{5} g_{6} \mu_{V}\right) \lambda_{D V}^{* *}} \\
& +\left(\Lambda_{H} \mu_{H} g_{3} g_{4} g_{5} g_{6}+\left(\Lambda_{H} \mu_{H} g_{4} g_{5} g_{6}+\left(\Lambda_{H} \gamma_{1} \mu_{H}+\tau_{1} \gamma_{1} \Lambda_{H}\right) g_{5} g_{6}\right) \lambda_{D V}^{* *}\right) \lambda_{D H}^{* *}
\end{aligned}
$$

substituting $\lambda_{D H}^{* *}$ to $\lambda_{D V}^{* *}$ we have

$$
A_{1} \lambda_{D v}^{* * 2}+A_{2} \lambda_{D v}^{* *}+A_{3}=0
$$

where

$$
\begin{aligned}
& A_{1}=\left(\mu_{H} g_{4}+\gamma_{1} \mu_{H}+\tau_{1} \gamma_{1}\right) \Lambda_{H} \mu_{H}^{2} g_{4} g_{5} g_{6}+g_{5} g_{6} \mu_{v}\left(\mu_{H} g_{4}+\gamma_{1} \mu_{H}+\tau_{1} \gamma_{1}\right)\left(\Lambda_{H} \gamma_{1} \mu_{H}+\tau_{1} \gamma_{1} \Lambda_{H}\right) \\
& +\beta_{H v} \mu_{H}^{2}\left(g_{4} \eta_{A}+\gamma_{1} \eta_{B}\right) \Lambda_{H} g_{4} g_{5} g_{6}+\beta_{H v} \mu_{H}\left(g_{4} \eta_{A}+\gamma_{1} \eta_{B}\right) g_{5} g_{6}\left(\Lambda_{H} \gamma_{1} \mu_{H}+\tau_{1} \gamma_{1} \Lambda_{H}\right) \\
& A_{2}=\left(\mu_{H} g_{4}+\gamma_{1} \mu_{H}+\tau_{1} \gamma_{1}\right) \Lambda_{H} g_{3} g_{4} g_{5} g_{6} \mu_{v}+\Lambda_{H} \mu_{H}^{3} g_{3} g_{4}^{2} g_{5} g_{6}+ \\
& \mu_{H} g_{3} g_{4} g_{5} g_{6} \mu_{v}\left(\Lambda_{H} \gamma_{1} \mu_{H}+\tau_{1} \gamma_{1} \Lambda_{H}\right)+\beta_{H v} \mu_{H}^{2}\left(g_{4} \eta_{A}+\gamma_{1} \eta_{B}\right) \Lambda_{H} \\
& g_{3} g_{4} g_{5} g_{6}-\beta_{H v} \beta_{v H} \Lambda_{H} \mu_{H}^{2} g_{3} g_{4}\left(\eta_{v} g_{6}+\gamma_{v}\right)\left(g_{4} \eta_{A}+\gamma_{1} \eta_{B}\right) \text {, }
\end{aligned}
$$




$$
\begin{aligned}
A_{3} & =\Lambda_{H} \mu_{H} g_{3}^{2} g_{4}^{2} g_{5} g_{6} \mu_{v}-\beta_{H v} \beta_{v H} \Lambda_{v} \mu_{H}^{2} g_{3} g_{4}\left(\eta_{v} g_{6}+\gamma_{v}\right)\left(g_{4} \eta_{A}+\gamma_{1} \eta_{B}\right) \\
& =\Lambda_{H} \mu_{H} g_{3}^{2} g_{4}^{2} g_{5} g_{6} \mu_{v}\left[1-\frac{\Lambda_{v} \beta_{H v} \beta_{v H} \mu_{H}\left(\eta_{v} g_{6}+\gamma_{v}\right)\left(g_{4} \eta_{A}+\gamma_{1} \eta_{B}\right)}{\Lambda_{H} g_{3} g_{4} g_{5} g_{6} \mu_{v}}\right] \\
A_{3} & =\Lambda_{H} \mu_{H} g_{3}^{2} g_{4}^{2} g_{5} g_{6} \mu_{v}\left[1-\left(R_{D}\right)^{2}\right]
\end{aligned}
$$

where $g_{3}=\mu_{H}+\gamma_{1}, g_{4}=\tau_{1}+\mu_{H}+\delta_{D 1}, g_{5}=\gamma_{1}+\mu_{v} g_{6}=\mu_{v}+\delta_{H v}$

Hence, we now claim the following

Theorem 1: The Dengue model (1) has a unique positive equilibrium if $R_{D}>1$.

Bifurcation Analysis of the model: Theorem 2: The model (1) undergoes backward bifurcation phenomenon at $R_{D}=1$ under certain condition.

Proof: The proof is based on the Centre manifold Theorem.

Let $x_{1}=S_{H}, x_{2}=E_{1}, x_{3}=I_{1}, x_{4}=R_{1}, x_{5}=S_{V}, x_{6}=E_{V}, x_{7}=I_{V}$. Further, let $\hat{f}=\left[f_{1}, \cdots, f_{7}\right]^{T}$ denote the vector field of the model (1). Thus, the model (1) can be written as:

$$
\begin{aligned}
\frac{d x_{1}}{d t} & =\Lambda_{H}-\mu_{H} x_{1}-\frac{\beta_{V H}\left(\eta_{V} x_{6}+x_{7}\right) x_{1}}{x_{1}+x_{2}+x_{3}+x_{4}}, \\
\frac{d x_{2}}{d t} & =\frac{\beta_{V H}\left(\eta_{V} x_{6}+x_{7}\right) x_{1}}{x_{1}+x_{2}+x_{3}+x_{4}}-\left(\gamma_{1}+\mu_{H}\right) x_{2}, \\
\frac{d x_{3}}{d t} & =\gamma_{1} x_{2}-\left(\tau_{1}+\mu_{H}+\delta_{D I}\right) x_{3}, \\
\frac{d x_{4}}{d t} & =\tau_{1} x_{3}-\mu_{H} x_{4}, \\
\frac{d x_{5}}{d t} & =\Lambda_{V}-\frac{\beta_{H V}\left(\eta_{A} x_{2}+\eta_{B} x_{3}\right) x_{5}}{x_{1}+x_{2}+x_{3}+x_{4}}-\mu_{V} x_{5}, \\
\frac{d x_{6}}{d t} & =\frac{\beta_{H V}\left(\eta_{A} x_{2}+\eta_{B} x_{3}\right) x_{5}}{x_{1}+x_{2}+x_{3}+x_{4}}-\left(\gamma_{V}+\mu_{V}\right) x_{6}, \\
\frac{d x_{7}}{d t} & =\gamma_{V} x_{6}-\left(\mu_{V}+\delta_{H V}\right) x_{7},
\end{aligned}
$$

The Jacobian of the transformed system (39), evaluated at the $D F E$, is given by: 
$J\left(\xi_{2}\right)=\left[\begin{array}{ccccccc}-\mu_{H} & 0 & 0 & 0 & 0 & -\beta_{v H}^{*} \eta_{v} & -\beta_{v_{H}}^{*} \\ 0 & -g_{3} & 0 & 0 & 0 & \beta_{v H}^{*} \eta_{v} & \beta_{v_{H}}^{*} \\ 0 & \gamma_{1} & -g_{4} & 0 & 0 & 0 & 0 \\ 0 & 0 & \tau_{1} & -\mu_{H} & 0 & 0 & 0 \\ 0 & 0 & 0 & 0 & -\mu_{V} & 0 & 0 \\ 0 & \beta_{v H}^{*} \eta_{A} x_{5}^{*} & \beta_{v H}^{*} \eta_{B} x_{5}^{*} & 0 & 0 & -g_{5} & 0 \\ 0 & 0 & 0 & 0 & 0 & \gamma_{v} & -g_{6}\end{array}\right]$,

40)

Suppose $\beta_{V H}=\beta_{V H}^{*}$ is chosen as the bifurcation parameter at $R_{D}=1$, we have that $\beta_{V H}^{*}=\frac{\Lambda_{H} g_{3} g_{4} g_{5} g_{6} \mu_{V}}{\Lambda_{V} \beta_{H V} \mu_{H}\left(g_{4} \eta_{A}+\gamma_{1} \eta_{B}\right)\left(\gamma_{V}+g_{6} \eta_{V}\right)}$

The right eigenvector of $J\left(\xi_{2}\right)_{\beta_{V H}=\beta_{V H}^{*}}$ is given by

$$
\begin{aligned}
& w=\left(w_{1}, w_{2}, w_{3}, w_{4}, w_{5}, w_{6}, w_{7}\right) \text { where, } \\
& w_{1}=-\frac{\beta_{H v} \beta_{v H}^{*} x_{5}^{*} w_{3}\left(\eta_{A}+\gamma_{1} \eta_{B}\right)}{\gamma_{1} g_{5} \mu_{H}}<0, w_{2}=\frac{w_{3}}{\gamma_{1}}, \\
& w_{3}=w_{3}>0 \\
& w_{4}=\frac{\tau_{1} w_{3}}{\mu_{H}}, w_{5}=0, w_{6}=\frac{\beta_{H v} x_{5}^{*} w_{3}\left(\eta_{A}+\gamma_{1} \eta_{B}\right)}{\gamma_{1} g_{5}} \\
& w_{7}=\frac{\beta_{H v} x_{5}^{*} \gamma_{v} w_{3}\left(\eta_{A}+\gamma_{1} \eta_{B}\right)}{\gamma_{1} g_{5} g_{6}}
\end{aligned}
$$

The above right eigenvector were obtained by solving (42) below.

$-\mu_{H} w_{1}-\beta_{v H}^{*} \eta_{v} w_{5}-\beta_{v H}^{*} w_{6}=0$

$-g_{3} w_{2}+\beta_{v H}^{*} \eta_{v} w_{5}+\beta_{v H}^{*} \eta_{v} w_{6}=0$,

$\gamma_{1} w_{2}-g_{4} w_{3}=0$

$\tau_{1} w_{3}-\mu_{H} w_{4}=0$

$-\mu_{v} w_{5}=0$,

$\beta_{H v} \eta_{A} x_{5}^{*} w_{2}+\beta_{H v} \eta_{B} x_{5}^{*} w_{3}-g_{5} w_{6}=0$

$\gamma_{v} w_{6}-g_{6} w_{7}=0$

Similarly, $J\left(\xi_{2}\right)_{\beta_{V H}=\beta_{V H}^{*}}$ has a left eigenvector, $v=\left(v_{1}, v_{2}, v_{3}, v_{4}, v_{5}, v_{6}, v_{7}\right)$ where, 


$$
\begin{aligned}
& v_{1}=0, v_{2}=\left(\gamma_{1} \eta_{B}+\eta_{A} g_{4}\right) \frac{v_{3}}{\eta_{B} g_{3}}, \\
& v_{3}=v_{3}>0, v_{4}=0, v_{5}=0 \\
& v_{6}=\frac{g_{3} v_{3}}{\beta_{H v} \eta_{B} x_{5}^{*}}, v_{7}=\frac{\beta_{v H}^{*}\left(\gamma_{1} \eta_{B}+\eta_{A} g_{4}\right) v_{3}}{\eta_{B} g_{3} g_{6}}
\end{aligned}
$$

The above eigenvectors were obtained by solving (44) below.

$$
\begin{aligned}
& -\mu_{H} v_{1}=0, \\
& -g_{3} v_{2}+\gamma_{1} v_{3}+\beta_{H v} \eta_{A} x_{5}^{*} v_{6}=0, \\
& -g_{4} v_{3}+\tau_{1} v_{4}+\beta_{H v} \eta_{B} x_{5}^{*} v_{6}=0, \\
& -\mu_{H} v_{4}=0, \\
& -\mu_{H} v_{5}=0, \\
& -\beta_{v H}^{*} \mu_{v} v_{1}+\beta_{v H}^{*} \eta_{v} v_{2}-g_{3} v_{6}+\gamma_{v} v_{3} v_{7}=0, \\
& -\beta_{v H}^{*} v_{1}+\beta_{v H} v_{2}-g_{6} v_{7}=0 .
\end{aligned}
$$

Computation of the bifurcation coefficient $a$ and $b$ for the Model: For the system (39), the associated non-zero partial derivatives required for the calculation of the backward bifurcation coefficients are given by

$$
\begin{aligned}
& \frac{\partial^{2} f_{2}}{\partial x_{2} \partial x_{6}}=\frac{\partial^{2} f_{2}}{\partial x_{6} \partial x_{2}}=\frac{-\beta_{V H}^{*} \eta_{V} \mu_{H}}{\Lambda_{H}}, \\
& \frac{\partial^{2} f_{2}}{\partial x_{2} \partial x_{7}}=\frac{\partial^{2} f_{2}}{\partial x_{7} \partial x_{2}}=\frac{-\beta_{V H}^{*} \mu_{H}}{\Lambda_{H}}, \\
& \frac{\partial^{2} f_{2}}{\partial x_{3} \partial x_{6}}=\frac{\partial^{2} f_{2}}{\partial x_{6} \partial x_{3}}=\frac{-\beta_{V H}^{*} \eta_{V} \mu_{H}}{\Lambda_{H}}, \\
& \frac{\partial^{2} f_{2}}{\partial x_{3} \partial x_{7}}=\frac{\partial^{2} f_{2}}{\partial x_{7} \partial x_{3}}=\frac{-\beta_{V H}^{*} \mu_{H}}{\Lambda_{H}}, \\
& \frac{\partial^{2} f_{2}}{\partial x_{4} \partial x_{6}}=\frac{\partial^{2} f_{2}}{\partial x_{6} \partial x_{4}}=\frac{-\beta_{V H}^{*} \eta_{V} \mu_{H}}{\Lambda_{H}}, \\
& \frac{\partial^{2} f_{2}}{\partial x_{4} \partial x_{7}}=\frac{\partial^{2} f_{2}}{\partial x_{7} \partial x_{4}}=\frac{-\beta_{V H}^{*} \mu_{H}}{\Lambda_{H}}, \\
& \frac{\partial^{2} f_{6}}{\partial x_{1} \partial x_{2}}=\frac{\partial^{2} f_{6}}{\partial x_{2} \partial x_{1}}=\frac{-\beta_{V H} \eta_{A} \mu_{H}^{2} \Lambda_{V}}{\Lambda_{H}^{2} \mu_{V}}, \\
& \frac{\partial^{2} f_{6}}{\partial x_{1} \partial x_{3}}=\frac{\partial^{2} f_{6}}{\partial x_{3} \partial x_{1}}=\frac{-\beta_{V H} \eta_{B} \mu_{H}^{2} \Lambda_{V}}{\Lambda_{H}^{2} \mu_{V}}, \\
& \frac{\partial^{2} f_{6}}{\partial x_{2}^{2}}=\frac{-2 \beta_{H V} \eta_{A} \mu_{H}^{2} \Lambda_{V}}{\Lambda_{H}^{2}}, \mu_{V}
\end{aligned}
$$




$$
\begin{aligned}
& \frac{\partial^{2} f_{6}}{\partial x_{2} \partial x_{3}}=\frac{\partial^{2} f_{6}}{\partial x_{3} \partial x_{2}}=\frac{-\beta_{V H} \mu_{H}^{2} \Lambda_{V}\left(\eta_{A}+\eta_{B}\right)}{\Lambda_{H}^{2} \mu_{V}}, \\
& \frac{\partial^{2} f_{6}}{\partial x_{2} \partial x_{4}}=\frac{\partial^{2} f_{6}}{\partial x_{4} \partial x_{2}}=\frac{-\beta_{V H} \eta_{B} \mu_{H}^{2} \Lambda_{V}}{\Lambda_{H}^{2} \mu_{V}}, \\
& \frac{\partial^{2} f_{6}}{\partial x_{3}^{2}}=\frac{-2 \beta_{H V} \eta_{B} \mu_{H}^{2} \Lambda_{V}}{\Lambda_{H}^{2} \mu_{V}}, \\
& \frac{\partial^{2} f_{6}}{\partial x_{3} \partial x_{4}}=\frac{\partial^{2} f_{6}}{\partial x_{4} \partial x_{3}}=\frac{-\beta_{H V} \eta_{B} \mu_{H}^{2} \Lambda_{V}}{\Lambda_{H}^{2} \mu_{V}}, \\
& \frac{\partial^{2} f_{2}}{\partial x_{6} \partial \beta_{V H}^{*}}=\eta_{V}, \\
& \frac{\partial^{2} f_{2}}{\partial x_{7} \partial \beta_{V H}^{*}}=1 .
\end{aligned}
$$

Since we know that,

$$
\begin{aligned}
& a=\sum_{k, \delta, j=1}^{7} v_{k} w_{i} w_{j} \frac{\partial^{2} f_{k}}{\partial x_{i} \partial x_{j}}(0,0), \\
& b=\sum_{k, i=1}^{7} v_{k} w_{i} \frac{\partial^{2} f_{k}}{\partial x_{i} \partial \beta_{v H}^{*}}(0,0),
\end{aligned}
$$

we now have that,

$$
\begin{aligned}
& a=2 v_{2} w_{2} w_{6} \frac{\partial^{2} f_{2}}{\partial x_{2} \partial x_{6}}(0,0),+2 v_{2} w_{2} w_{7} \frac{\partial^{2} f_{2}}{\partial x_{2} \partial x_{7}}(0,0)+2 v_{2} w_{3} w_{6} \frac{\partial^{2} f_{2}}{\partial x_{3} \partial x_{6}}(0,0) \\
& +2 v_{2} w_{2} w_{7} \frac{\partial^{2} f_{2}}{\partial x_{3} \partial x_{7}}(0,0)+2 v_{2} w_{4} w_{6} \frac{\partial^{2} f_{2}}{\partial x_{4} \partial x_{6}}(0,0)+2 v_{2} w_{4} w_{7} \frac{\partial^{2} f_{2}}{\partial x_{4} \partial x_{7}}(0,0) \\
& +2 v_{6} w_{1} w_{2} \frac{\partial^{2} f_{6}}{\partial x_{1} \partial x_{2}}(0,0)+2 v_{6} w_{1} w_{2} \frac{\partial^{2} f_{6}}{\partial x_{1} \partial x_{3}}(0,0)+v_{2} w_{2}{ }^{2} \frac{\partial^{2} f_{6}}{\partial x_{2}{ }^{2}}(0,0) \\
& +2 v_{6} w_{2} w_{3} \frac{\partial^{2} f_{6}}{\partial x_{2} \partial x_{3}}(0,0)+2 v_{6} w_{2} w_{4} \frac{\partial^{2} f_{6}}{\partial x_{2} \partial x_{4}}(0,0)+v_{6} w_{3}{ }^{2} \frac{\partial^{2} f_{6}}{\partial x_{3}{ }^{2}}(0,0)+2 v_{6} w_{3} w_{4} \frac{\partial^{2} f_{6}}{\partial x_{3} \partial x_{4}}(0,0), \\
& \text { which leads to }
\end{aligned}
$$

$$
\begin{aligned}
& a=K_{1}-\left(K_{2}+K_{3}\right), \text { where, } \\
& \mathrm{K}_{1}=\frac{2 g_{3} g_{4}^{2} g_{6} \nu_{3} w_{3}\left(\eta_{A}+\gamma_{1} \eta_{B}\right)}{\Lambda_{H} \gamma_{1}\left(g_{4} \eta_{A}+\gamma_{1} \eta_{B}\right)\left(\gamma_{V}+g_{6} \eta_{V}\right)}\left[\frac{\eta_{A}}{\eta_{B}}+w_{3}\right], \\
& \mathrm{K}_{2}=\frac{2 g_{4} g_{6} v_{3} w_{3}^{2}\left(\eta_{A}+\gamma_{1} \eta_{B}\right)}{\eta_{B} \gamma_{1} \mu_{H}\left(\gamma_{V}+g_{6} \eta_{V}\right)}\left[\mu_{H}\left[\frac{1}{\gamma_{V}}\left(\eta_{V}+\gamma_{V}\right)+\eta_{V}+\frac{\gamma_{V}}{g_{6}}\right]+\tau_{1}\left(\eta_{V}+\frac{\gamma_{V}}{g_{6}}\right)\right],
\end{aligned}
$$




$$
\mathrm{K}_{3}=\frac{2 g_{4} v_{3} w_{3}^{2} \mu_{H}}{\Lambda_{H}^{2}}\left[\frac{1}{\eta_{B} \gamma_{1}}\left(\frac{\eta_{A} \mu_{H}}{\gamma_{1}}+\eta_{A}+\eta_{B}+\tau_{1} \eta_{A}\right)+1+\tau_{1}\right]
$$

And

$b=\sum_{k, i=1}^{7} v_{k} w_{i} \frac{\partial^{2} f_{k}}{\partial x_{i} \partial \beta_{v H}^{*}}(0,0)$,

Also we have that,

$$
\begin{aligned}
& b=v_{2} w_{6} \frac{\partial^{2} f_{2}}{\partial x_{6} \partial \beta_{v H}^{*}}(0,0)+v_{2} w_{7} \frac{\partial^{2} f_{2}}{\partial x_{7} \partial \beta_{v H}^{*}}(0,0) \\
& b=\frac{\left(\gamma_{1} \eta_{B}+\eta_{A} g_{4}\right)\left(\eta_{A}+\gamma_{1} \eta_{B}\right) \beta_{H v} x_{5}^{*} w_{3}}{\eta_{B} g_{3} g_{5} \gamma_{1}}\left[\eta_{v}+\frac{\gamma_{v}}{g_{6}}\right] .
\end{aligned}
$$

It follows from (49) that the bifurcation coefficient, $a$, is positive whenever, $K_{1}>K_{2}+K_{3}$

Thus, the model (1) undergoes a backward bifurcation at $R_{D}=1$ whenever the inequality (55) holds.

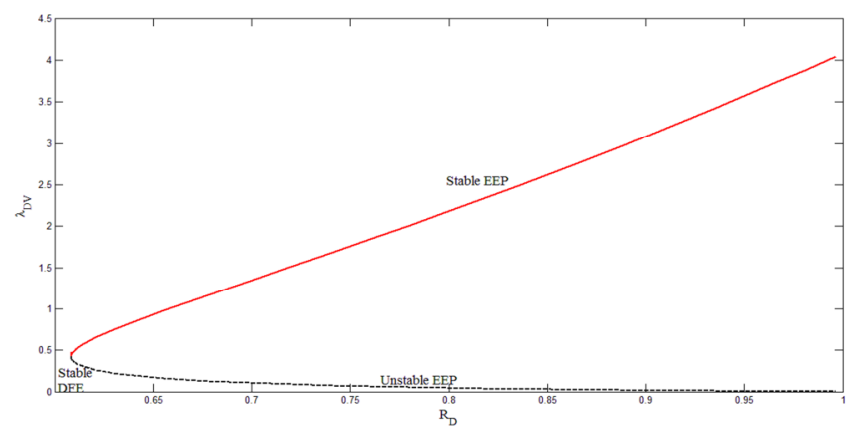

Fig. 3: Backward bifurcation diagram for the model (1) showing the force of infection $\lambda_{D V}$ as a function of the control reproduction number $R_{D}$ with all the parameters used as stated in Table (2) except $\beta_{V H}=2$ and $\beta_{H V}=1$ so that $R_{D}<1$.

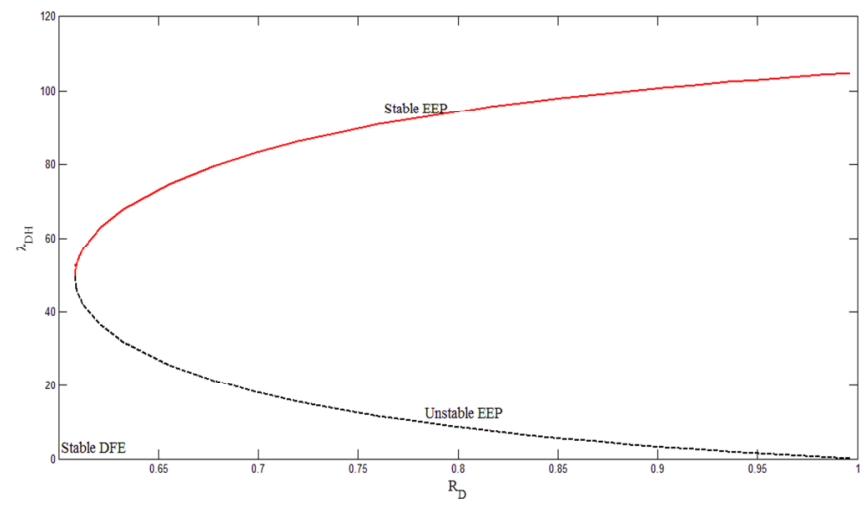


Fig. 4: Backward bifurcation diagram for the model (1) showing the force of infection $\lambda_{D H}$ as a function of the control reproduction number $R_{D}$ with all the parameters used as stated in Table (2) except $\beta_{V H}=2$ and $\beta_{H V}=1$ so that $R_{D}<1$.

\section{Simulations}

Table 3: Parameter Information Using the parameter values in Table 3, we carried out some simulations of

\begin{tabular}{clll} 
& \multicolumn{3}{c}{ model (1). } \\
\hline Parameter & Values & Unit & References \\
\hline $\boldsymbol{\Lambda}_{\boldsymbol{H}}$ & 500 & Year $^{-1}$ & Garba et al, 2008. \\
$\boldsymbol{\Lambda}_{\boldsymbol{V}}$ & $10^{7}$ & Year $^{-1}$ & Garba et al, 2008. \\
$\boldsymbol{\mu}_{\boldsymbol{H}}$ & O.O2041 & Year $^{-1}$ & Okuonghae and Omosigho (2011) \\
$\boldsymbol{\mu}_{\boldsymbol{V}}$ & 36.5 & Year $^{-1}$ & Okuonghae and Omosigho (2011) \\
$\boldsymbol{\beta}_{\boldsymbol{V} \boldsymbol{H}}$ & 5 & Year $^{-1}$ & Garba et al, 2008. \\
$\boldsymbol{\beta}_{\boldsymbol{H} \boldsymbol{V}}$ & 4 & Year- $^{-1}$ & Gubler,1998 \\
$\boldsymbol{\tau}_{\mathbf{1}}$ & 2.5 & Ind $^{-1}$ Year $^{-1}$ & Garba et al, 2008. \\
$\boldsymbol{\gamma}_{\mathbf{1}}$ & 0.3254 & Year $^{-1}$ & Gubler, 1998 \\
$\boldsymbol{\gamma}_{\boldsymbol{V}}$ & 0.03 & Year $^{-1}$ & Garba et al, 2008. \\
& & & \\
$\boldsymbol{\delta}_{\boldsymbol{D} \mathbf{1}}$ & 0.365 & Year $^{-1}$ & Gubler,1998 \\
$\boldsymbol{\delta}_{\boldsymbol{H} \boldsymbol{V}}$ & 0 & Year $^{-1}$ & Gubler,1998 \\
$\boldsymbol{K}_{\boldsymbol{V}}$ & 0.02 & Year $^{-1}$ & Garba et al, 2008. \\
$\boldsymbol{\eta}_{\boldsymbol{i}}(\boldsymbol{i}=\boldsymbol{A}, \boldsymbol{B})$ & $0.6,1$ & Year $^{-1}$ & Garba et al, 2008. \\
$\boldsymbol{\eta}_{\boldsymbol{V}}$ & 0.5 & Year $^{-1}$ & Garba et al, 2008. \\
\hline
\end{tabular}

\section{RESULTS AND DISCUSSION}

Biologically speaking, Dengue is eliminated from the population when $R_{D}<1$ if the initial sizes of the populations of the model are in the region of attraction of $\xi_{2}$. However, the disease free equilibrium may not be globally asymptotically stable even if $R_{D}<1$ in the case when a backward bifurcation occurs. That is, there is the presence of a stable EEP co-existing with the DFE. The model undergoes the phenomenon of backward bifurcation at $R_{D}=1$ whenever the inequality (55) holds.

Conclusion: In this paper, a mathematical model is proposed and analyzed to study the transmission dynamics of Dengue fever in a human population with treatment. Analyzing the models revealed that: The model undergoes a phenomenon of backward bifurcation if a certain condition shown in inequality (55) holds. The model possesses the disease free equilibrium and it also has an endemic equilibrium. Finally, the results from the numerical simulations show that treatment is crucial for an effective public health control of dengue fever.

\section{REFERENCES}

Gubler, DJ (1998). Dengue and Dengue Hemorrhagic Fever, Clin Microbial. Rev 480-496,

Gubler DJ; Edward B (1981). Dengue and Dengue Haemorhagic Fever, $C D C$ wonder
Garba, SM; Gumel, AB; Abubakar; MR (2008). Backward Bifurcations in Dengue Transmission Dynamics. Mathematical Bioseiences, 215:11-25

Nuraini, N; Tasman, HE; Soewono, K A; Sidarto, (2009). A with-in Host Dengue Infection Model with Immune Response. Maths and Computer Modelling, 49 1148-1155

Okuonghae, D.; Omosigho SE (2011). Analysis of Mathematical Model for Tuberculosis. Journal of Theo. Biol., 269:31-45

Van Den Driessche; Watmough, J. (2002). Reproductive Numbers and Sub-threshold Endemic Equilibria for Compartment Models of Disease Transmission. J. Math. Biol, 180 2948.

Vaughn, DW, Hoke CH, Yoksan S, et al., (1996). Testing of a dengue 2 live-attenuated vaccine (strain 16881 PDK 53) in ten American volunteers. Vaccine, 14:329-336. 